\title{
Lost treasures: a plea for the systematic preservation of cadaveric heart specimens through three-dimensional digital imaging
}

\author{
Stephen P. Seslar, ${ }^{1}$ Charles W. Shepard,${ }^{2}$ Jorge M. Giroud, ${ }^{3}$ Vera D. Aiello, ${ }^{4}$ Andrew C. Cook, ${ }^{5}$ \\ Diane E. Spicer, ${ }^{6,7}$ James D. St. Louis, ${ }^{8}$ Rodney C.G. Franklin, ${ }^{9}$ Jeffrey P. Jacobs, ${ }^{10,11}$ Robert H. Anderson, ${ }^{12}$ \\ On behalf of the Archiving Working Group of The International Society for Nomenclature of Paediatric \\ and Congenital Heart Disease ${ }^{13}$
}

${ }^{1}$ Department of Pediatrics, Division of Pediatric Cardiology, Seattle Children's Hospital, University of Washington, Seattle, Washington; ${ }^{2}$ Department of Pediatrics, Division of Pediatric Cardiology, University of Minnesota Children's Hospital, University of Minnesota, Minneapolis, Minnesota; ${ }^{3}$ All Children's Hospital, The Congenital Heart Institute of Florida, Pediatric Cardiology Associates/Pediatrix Medical Group, Saint Petersburg, Florida, United States of America; ${ }^{4}$ Laboratory of Pathology, Heart Institute (InCor), University of Sao Paulo Medical School, Sao Paulo, Brazil; ${ }^{5}$ Cardiac Unit, Institute of Child Health, University College London, London, United Kingdom; 'Johns Hopkins All Children's Heart Institute, All Children's Hospital and Florida Hospital for Children, Saint Petersburg, Tampa, and Orlando, Florida, United States of America; ${ }^{7}$ University of Florida, Department of Pediatric Cardiology, Gainesville, Florida, United States of America; ${ }^{8}$ Department of Surgery, Children's Mercy Hospital, Kansas City, Missouri; ${ }^{9}$ Paediatric Cardiology Directorate, Royal Brompton \& Harefield NHS Trust, Harefield, Middlesex, United Kingdom; ${ }^{10}$ Division of Cardiovascular Surgery, Department of Surgery, Johns Hopkins All Children's Heart Institute, All Children's Hospital and Florida Hospital for Children, Saint Petersburg, Tampa, Orlando, Florida, United States of America; ${ }^{11}$ Division of Cardiac Surgery, Department of Surgery, Johns Hopkins University, Baltimore, Maryland, United States of America; ${ }^{12}$ Institute of Genetic Medicine, Newcastle University, Newcastle upon Tyne, United Kingdom; ${ }^{13}$ www.ipccc.net

S INCE THE BIRTH OF PAEDIATRIC AND CONGENITAL cardiac care, and indeed preceding it, those of us practising in this field have benefited from an indispensable and, in its present form, irreplaceable resource for learning about the patients for whom we care. From the generous gifts of patients and their families, meticulously curated by a handful of individuals over our field's 65-year history, we have cultivated exceptional collections of cadaveric heart specimens, exceeding in some cases 25,000 specimens. Although preservation in formalin, wax, or by plastination has remarkably slowed the natural degradation process of the specimens, these processes do not render time powerless. The pioneer collections, such as the Osler and Abbott collections in the Maude Abbott Medical Museum at McGill University in Montreal, Canada, began in the late

Correspondence to: S. P. Seslar, MD, PhD, Department of Pediatrics, Division of Pediatric Cardiology, Seattle Children's Hospital, 4800 Sand Point Way NE, M/S RC.2.820, Seattle, WA 98105, United States of America. Tel: 206987 6993; Fax: 206987 3839; E-mail: stephen.seslar@seattlechildrens.org 19th century. The compilation established by Maurice Lev dates from the 1940s, and was assembled from several institutions in Chicago. Most of the large collections were started in the 1960s and 1970s, with the pace of curation being the greatest through the 1980 s and 1990s. This means that the bulk of the specimens in these collections has now aged in excess of 30-40 years, with some unique specimens being over 75 years old. Owing to the inexorable forces of time, and the hands of countless teachers and trainees that have pored over the specimens, these rare gems are quite literally disintegrating before our eyes. Despite preservatives and loving care, it has been estimated that, in some collections, more than half the hearts have already been rendered useless, and thus lost - forever. The time has come to stop the loss of these treasures. The technology to do just that is now readily available.

Technologies such as three-dimensional scanning and printing have been available since the early 1990s. The process is used extensively in commercial manufacturing, where it is known as rapid prototyping. 
It is also widely used in archaeology and entertainment, as well as in the creation of orthotics and prosthesis. The value of this technology, nonetheless, has only recently been appreciated by the clinical medical community. Although there are a number of methods to create the end product, the process is conceptually quite simple; three-dimensional data - that is, $x, y$, and $z$ coordinates of the physical structure of a given item are fed from a computer to a specially designed printer, which literally "prints" the structure layer by layer, or creates a solid mould from a liquid precursor. The result is a precise replica of the original specimen (Fig $1 \mathrm{a}$ and $\mathrm{b}$ ). Through the wonders of material engineering, the resultant printed structures can have a wide range of physical characteristics including hard, soft, clear, coloured, and so on. Applying this technology to cadaveric heart specimens means that these treasures can literally be made immortal. Once digitally
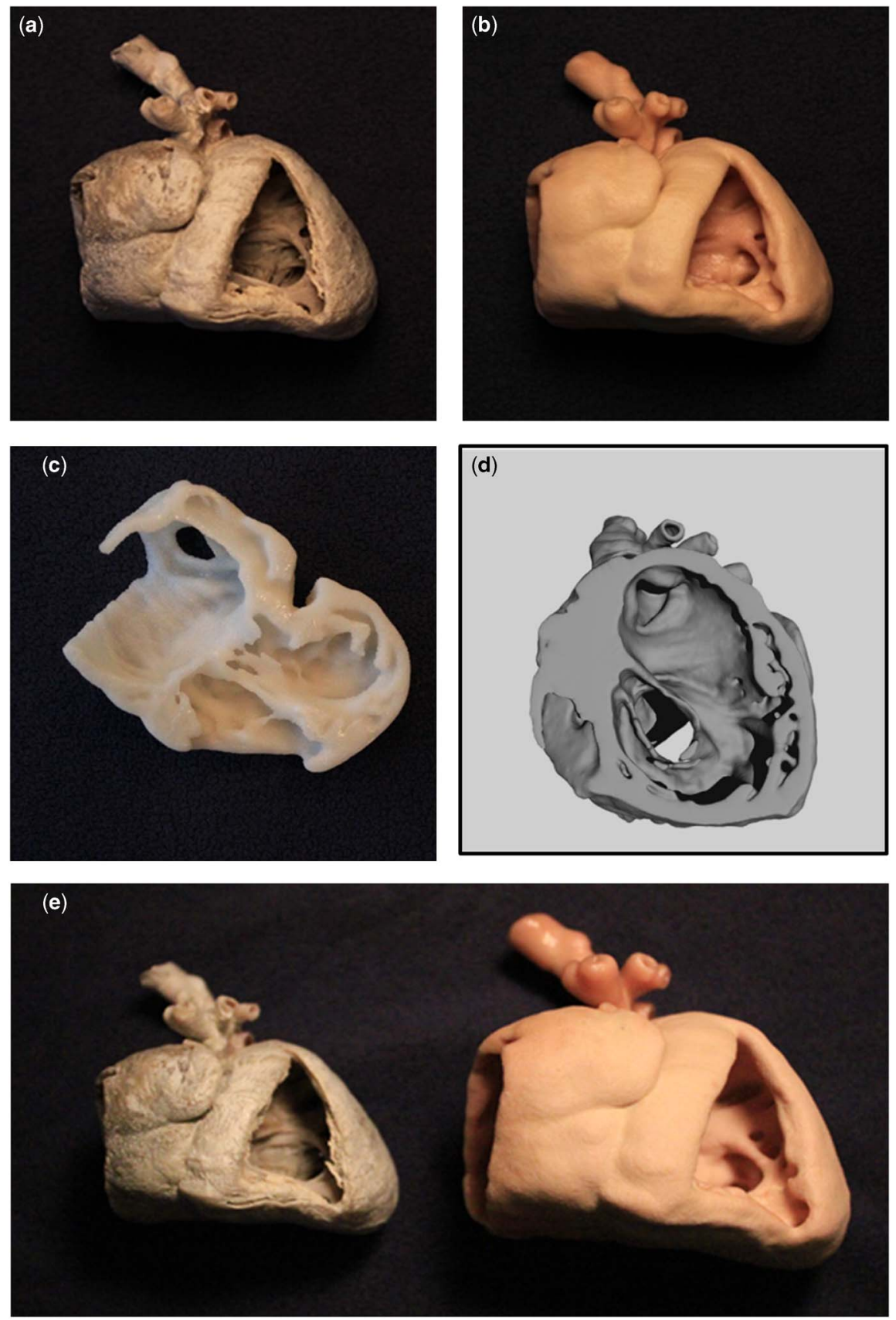

Figure 1.

Waxed heart specimen and three-dimensional reproductions from a CT scan. (a) Cadaveric waxed beart specimen from a patient with bypoplastic left heart syndrome; (b) three-dimensional printed replica of the waxed heart specimen; (c) three-dimensional printed model of digitally created four-chamber view; $(\boldsymbol{d})$ digital three-dimensional rendering of a short-axis segmentation; $(\boldsymbol{e})$ the original cadaveric specimen side-by-side with a three-dimensional replica printed at $1.5 \times$ the original size. 
Table 1. Suggestions for three-dimensional digital imaging of cadaveric heart specimens.

1. Entire heart should be imaged (superior to inferior caval vein, including the aortic arch when available)

2. Formalin-fixed specimens may be filled with cotton balls or placed in a gel suspension to restore a more natural or "filled" appearance

3. Once scanned, tissue structures of interest should be easily visible to the naked eye with good contrast compared with nearby tissue and with well-defined edges

4. Multiple- and independent-image data sets can be combined to make up for the deficiencies of a single-image data set

5. Out-of-plane image resolution should be no greater than two times the in-plane image resolution (e.g. pixel size of 0.5 , $0.5,1.0$ mm)

rendered using three-dimensional imaging, the resulting data set is, in many ways, even more valuable than the original specimen itself. The process of digitalisation frees the pathological specimen to be used for other purposes such as histopathological analysis. Thus, with digital reproduction, we can quite literally have our cake and eat it too. The process of digitalisation also allows the data to be utilised in a manner that best suits the needs of the educational goal for which it is being produced - for example, the same digital data set can be viewed on a monitor, holographically, in virtual laboratory settings, and printed as a three-dimensional model. Virtual "dissections" can be performed such that a heart model is created to illustrate a specific internal structure that would be impossible to see in the intact specimen (Fig 1c and d). Similarly, dissections of three-dimensional models can be performed in multiple planes, including standard echocardiographic planes such as the parasternal long-axis plane, the subcostal short-axis plane, and the apical four-chamber plane. Structures can be colour-coded to help students grasp physiology as well as anatomy. Small hearts can be enlarged to see the details easily missed in the original specimen (Fig 1e). Most importantly, when the model of a cadaveric specimen wears out after years of use, a precise replica can be simply and inexpensively reprinted.

Nevertheless, for all its elegant simplicity, rapid prototyping is of no use without the raw material. Although three-dimensional imaging of living patients will provide us an endless source of material for the creation of modern "living heart" registries, the cadaveric heart specimens amassed over the years by the early curators in our field represent an irreplaceable and priceless historic account of the birth and infancy of our specialty. There are specimens in these collections that, either because of their rarity in nature or because of their place in the evolution of our field, may never be seen again. With the ever-increasing capabilities of modern imaging systems, it may be tempting to put off the process of preserving cadaveric material through digital three-dimensional imaging until we each have the "next-generation" scanner. This is a recipe for inaction. Such procrastination will only lead to the loss of more raw materials. Owing to the lack of cardiac motion, even computerized axial tomography (CT) and magnetic resonance imaging (MRI) scanners that are relatively crude by today's standards can produce excellent reproductions (Fig 1). The manipulation of the data set produced also illustrates what can be achieved, as discussed above. We propose, therefore, along with our plea for action, a few simple recommendations for generating three-dimensional digital images of cadaveric heart specimens that are suitable for model creation (Table 1).

The benefits of digitalising pathological cardiovascular archives extend beyond longevity - namely, accessibility. The cadaveric specimens in all collections, big and small, represent the gift of patients and their families that asked for nothing more in return apart from the assurance that these precious specimens would be used to their fullest potential to improve the lives of future generations of those living with congenital heart disease. With open sourcing, the portability of digital data, and the evermore ubiquitous availability of three-dimensional printers, the creation of digital versions of these collections will allow the teachers of congenital cardiology and their students to produce the models anywhere they happen to be. The Archiving Working Group of the International Society of Nomenclature for Paediatric and Congenital Heart Disease is presently creating a digital reference library of congenital cardiac disease, including photographs of pathological specimens, echocardiographic clips, and CT and MRI images. This forum will similarly function as a repository for three-dimensional data sets that can be used for the creation of cardiac models.

The heart is a wonderfully complex geometric structure that is difficult to understand and appreciate fully when represented in only two dimensions. Even digital three-dimensional renderings, when displayed in two-dimensional format, do not convey the information contained in a physical threedimensional structure that can be grasped and manipulated in one's own hand. Patients and their families have provided us with remarkable resources to improve our understanding of the patients for whom we care. The pathologists and anatomists have lovingly and painstakingly curated these gifts into irreplaceable collections. To achieve their true potential, it is now up to us to make these collections timeless through digital rendering. 